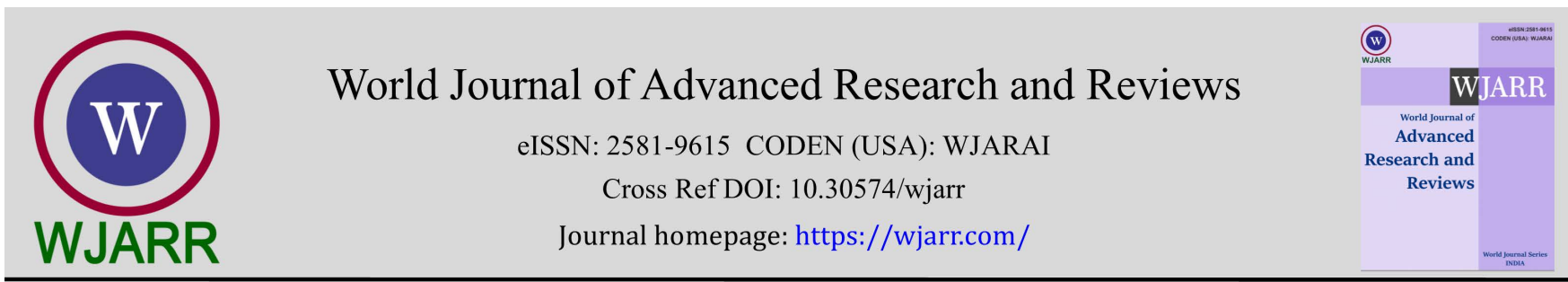

(RESEARCH ARTICLE)

\title{
Identification of a new coronavirus in rectal swabs from persons infected 15-25 days ago
}

\author{
Anna Liskova ${ }^{1}$ and Vladimir Zajac ${ }^{2, *}$ \\ ${ }^{1}$ Department of Clinical Microbiology, Spitálska 6, University Hospital Nitra, Slovakia. \\ ${ }^{2}$ Former scientist at the Cancer Research Institute, BMC, SAS, Bratislava, Dubravska cesta 9, 84505 Slovakia.
}

World Journal of Advanced Research and Reviews, 2021, 10(01), 356-359

Publication history: Received on 18 March 2021; revised on 25 April 2021; accepted on 27 April 2021

Article DOI: https://doi.org/10.30574/wjarr.2021.10.1.0179

\begin{abstract}
Every virus is a parasite that cannot exist on its own and is fully dependent on its carrier. This is the basic condition of its existence. The parasite must have its host, and thus is a living cell, but it is generally claimed that the virus can exist without a carrier for 2 to 5 seconds, during which it is transmitted to another species. Following these viral pathways is not easy and has not been fully investigated. However, this is a key issue that can lead to a major paradigm shift in the way we perceive viruses. The primary carrier, however, cannot be host organ cells because they could easily damage them. In terms of the mechanism of existence and transmission, the following could be the most suitable carriers of the virus: bacteria, yeast or other single-cell organisms.
\end{abstract}

Keywords: Novel coronavirus; RT PCR; Virus carriers; Rectal swabs; Intestinal tract; Fecal infection

\section{Introduction}

Based on work with bovine leukosis virus (BLV) in the stables, we monitored the course of infection in healthy animals and concluded that a bacterial cell can be the host the virus. We tested this assumption and confirmed the results. This idea was then tested on the HIV model. Even with this virus, we have been able to prove that its host may be bacteria. Evidence was confirmed at the DNA level by hybridization and PCR using commercial, diagnostic primers and consequent sequencing (1-11). At the protein level, HIV-like proteins were confirmed by using commercial monoclonal antibodies against HIV antigens. In the swab of HIV-positive children from Cambodia and Kenya, HIV was found in commensal bacteria (1-11), but it is also often found in the yeast Candida albicans.

After transmission to humans, the coronavirus travels to cells of the respiratory tract that contain the ACE2 receptor. Upon contact of viral tentacles with this receptor, the virus penetrates the receiving cell of the respiratory tract, where the process of tissue destruction occurs. After overcoming the infection and eliminating the virus in the recipient's lung cells by a conventional drug-based treatment approach such as ventilation and activation of the immune system, the infection is suppressed and the patient can be pronounced cured. However, the carriers containing the virus survive in the intestinal tract and can multiply under optimal conditions and they then penetrate the body. People after having been infected may become carriers of the coronavirus and thus may infect others by secreting the coronavirus in the form of droplets, but also in faeces. Surprisingly, no attention is currently being paid to a possible fecal infection. However, history provides us with a lot of evidence about the importance of disinfection and disposal of faeces during epidemics. This important approach has been proven many times in history and has helped to overcome epidemics. It is incomprehensible why, in the current epidemic, this possibility of transmitting infection is absolutely marginal and has not been considered. We have become better at successfully preventing the droplet aspect of this infection with face

\footnotetext{
${ }^{*}$ Corresponding author: Assoc. prof. RNDr. Vladimir Zajac, PhD

Former scientist at the Cancer Research Institute, BMC, SAS, Bratislava, Dubravska cesta 9, 84505 Slovakia currently an emeritus researcher.
} 
masks, social distancing and hand washing, however, but we are not yet sufficiently preventing rectal infection. It is necessary to introduce rapid disposal of excrement, disinfection of toilets and UV emitters, ventilation, careful washing of the rectum and finally washing and disinfection of hands. This must be done wherever there are shared toilets - social service homes, schools, workplaces, hospitals, airplanes, buses, but also in households. After all, it is this form of virus transmission that can answer the question about what may be the mechanism of induction of the second wave of the infection.

\section{Methodology}

Based on our more than 30 years of work with viruses (BLV, HIV), achieved results and inventions, rectal swabs were taken from persons who overcame the infection with a new coronavirus at the Institute of Clinical Microbiology at the University Hospital Nitra (Slovakia) under the leadership of prof. MUDr. Anna Líšková PhD.

\section{Results and Discussion}

Table 1 RT PCR of SARS CoV -2 from nasopharyngeal and rectal swabs from patients infected 15-25 days ago.

\begin{tabular}{|c|c|c|}
\hline & PCR SARS CoV -2 nasopharyngeal swab & PCR SARS CoV-2stool \\
\hline 1 & 3.3. 2021 PCR pozit. (Ct 26,7) & 26.3. 2021 PCR pozit. (Ct 34,8) \\
\hline 2 & 6.3. 2021 PCR pozit. (Ct 22) & 27.3. 2021 PCR pozit. (Ct 22,6) \\
\hline 3 & 12.3. 202 PCR pozit.( Ct 24,2) & 23.3. 2021 PCR pozit. (Ct 30,64) \\
\hline 4 & 3.3. 2021 PCR pozit. (Ct 22,0$)$ & 27.3. 2021 PCR pozit. (Ct 22,6) \\
\hline 5 & 9.3. 2021 PCR pozit. (Ct 24,8) & 26.3. 2021 PCR pozit. (Ct 31,0) \\
\hline 6 & 13.3. 2021 PCR pozit. (Ct 31,7) & 27.3. 2021 PCR pozit. (Ct 22,6) \\
\hline 7 & 26.2. 2021 PCR pozit.( Ct 28,8) & 26.3. 2021 PCR pozit. (Ct 31,0) \\
\hline 8 & 7.3. 2021 PCR pozit.(Ct 22,2) & 19.3. 2021 PCR pozit. (Ct 22,6) \\
\hline 9 & 10.3. 2021 PCR pozit. (Ct 18,2) & 29.3. 2021 PCR pozit. (Ct 27,8) \\
\hline 10 & 1.3. 2021 PCR pozit. (Ct 23,4$)$ & 27.3. 2021 PCR pozit. (Ct 27,4) \\
\hline 11 & 12.3. 2021 PCR pozit. (Ct 18,8 ) & 26.3. 2021 PCR pozit. (Ct 27,2) \\
\hline 12 & 9.3. 2021 PCR pozit. (Ct 28,9) & 19.3. 2021 PCR pozit. (Ct 21,6) \\
\hline 13 & 4.3. 2021 PCR pozit. (Ct 24,02) & 26.3. 2021 PCR pozit. (Ct 30,0) \\
\hline 14 & 7.3. 2021 PCR pozit. (Ct 26,6) & 27.3. 2021 PCR pozit. (Ct 22,4) \\
\hline 15 & 10.3. 2021 PCR pozit. (Ct 21,5) & 26.3. 2021 PCR pozit. (Ct 30,4) \\
\hline 16 & 8.3. 2021 PCR pozit. (Ct 20,0 ) & 27.3. 2021 PCR pozit. (Ct 28,5) \\
\hline 17 & 7.3. 2021 PCR pozit. (Ct 26,6$)$ & 27.3. 2021 PCR pozit. (Ct 27,0) \\
\hline 18 & 15.2. 2021 PCR pozit. (Ct 31,4) & 26.3. 2021 PCR negat. \\
\hline 19 & 10.3. 2021 PCR pozit. (Ct 24,3) & 29.3. 2021 PCR negat. \\
\hline 20 & 7.3. 2021 PCR pozit. (Ct 26,6) & 26.3. 2021 PCR pozit. (Ct 30,8) \\
\hline 21 & 8.3. 2021 PCR pozit. (Ct 27,5) & 30.3. 2021 PCR pozit. (Ct 32,2) \\
\hline 22 & 15.3. 2021 PCR pozit. (Ct 33,5) & 26.3. 2021 PCR negat. \\
\hline 23 & 12.3. 2021 PCR pozit. (Ct 31,56) & 27.3. 2021 PCR negat. \\
\hline 24 & 10.3. 2021 PCR pozit. (Ct 21,5 ) & 26.3. 2021 PCR pozit. (Ct 30,2) \\
\hline 25 & 12.3. 2021 PCR pozit. (Ct 20,2) & 27.3. 2021 PCR pozit. (Ct 28,5) \\
\hline
\end{tabular}


World Journal of Advanced Research and Reviews, 2021, 10(01), 356-359

\begin{tabular}{|l|l|l|}
\hline 26 & 11.3.2021 PCR pozit. (Ct 23,02) & 29.3. 2021 PCR pozit. (Ct 20,13) \\
\hline 27 & 12.3.2021 PCR pozit. (Ct 30,01) & 27.3. 2021 PCR negat. \\
\hline 28 & 7.3.2021 PCR pozit. (Ct 24,5) & 29.3. 2021 PCR pozit. (Ct 30,4) \\
\hline 29 & 7.3.2021 PCR pozit. (Ct 27,3) & 29.3. 2021 PCR pozit. (Ct 30,01) \\
\hline 30 & 12.3.2021 PCR pozit. (Ct 23,88) & 29.3. 2021 PCR pozit. (Ct 31,7) \\
\hline
\end{tabular}

The obtained results confirm that out of a cohort of 30 tested persons, only five patients $(17 \%)$ did not show signs of presence of the novel coronavirus in their stool more than 12-23 days after being infected, as proven by RT PCR tests. The remaining 25 subjects (83\%) still had presence of coronavírus in their stool more than 12-23 days after being infected. The viral load of nasal swabs detected by RT PCR tests was lower in four out of the five patients who tested negative using RT PCR from rectal swabs. The results show that the vast majority of patients who have overcome the infection, the virus is still present in the stool and their intestinal tract. This supports the assumption that these individuals may still be infectious and may pass the virus to other people. It is therefore recommended that these persons strictly follow the necessary hygiene measures as mentioned above. Strict adherence to the recommended measures is expected to radically reduce the number of newly infected people and victims.

\section{Conclusion}

The coronavirus has been found in the stools of infected people in several laboratories, but this explains that the virus leaves the body and clears itself of the virus. But that is not the case. The virus does not leave, remains in the body, is established there and can exist there in the carrier. Surprisingly, no one observed the virus in the intestinal tract at such an interval after infection. The detection of the novel coronavirus in the intestinal tract of people who overcame the infection 2-3 weeks before being tested raises a fundamental question: in what form does the virus exist in the tract? A virus such as a parasite cannot exist on its own, cannot reproduce and cannot be transmitted to another organism. It therefore must have a carrier. So, what carriers does the virus use to persist in the intestinal tract, but also throughout the body? Can they be bacteria or yeast? Or some other single-celled organisms? By identifying the carrier or carriers of the virus and their subsequent elimination, we also eliminate the virus. And that may bring the epidemic to its end.

\section{Compliance with ethical standards}

\section{Acknowledgments}

Author is grateful to L. Wachsmannova, S. Ciernikova, K. Hainova, Z. Adamcikova and V. Stevurkova for participation in this work. I am also grateful to $\mathrm{H}$. Komjathy for support and stimulating discussion. This work was supported by these grants: APPV-06-46-11, VEGA 2/0096/11 and VEGA 2/0170/13. This publication is also the result of the project implementation: SF ITMS project code: 26240220058 supported by the Research \& Development Operational Programme funded by the ERDF.

\section{Disclosure of conflict of interest}

The authors declare that there is no conflict of interest.

\section{Statement of informed consent}

Informed consent was obtained from all individual participants included in the study.

\section{References}

[1] V Zajac V, Kovac M, Ciernikova S, Mego M, Rauko P, Stevurkova V, Stanekova D, Mokras M. Detection of HIV sequences in colon bacteria of AIDS positive patients. Clin. Microbiol Infec. 2005; 11: 53.

[2] Zajac V, Mego M, Kovac M, Stevurkova V, Ciernikova S, Ujhazy E, Gajdosik A, Gajdosikova A. Testing of bacteria isolated from HIV/AIDS patients in Experimental models. Neuro Endocrinol Lett. 2006; 27: 101-104.

[3] Zajac V, Stevurkova V, Matelova L, Ujhazy E. Detection of HIV-1 sequences in intestinal bacteria of HIV/AIDS patients. Neuro Endocrinol Lett. 2007; 28: 591-595. 
[4] Zajac V, Adamcikova Z, Holec V, Hainova K, Stevurkova V, Matelova L, Krcmery V. Chapter in a book: Microbes, viruses and parasites in AIDS process". Editor: VladimirZajac. Printed in INTECH. 2011; 375-390.

[5] Zajac V, Matelova L, Liskova A, Mego M, Holec V, Adamcikova Z, Stevurkova V, Shahum A, Krcmery V. Confirmation of HIV-like sequences in respiratory tract bacteria of and HIV-positive pediatric patients. Med Sci Monit. 2011; 17: 154-158.

[6] Hainova K, Mego M, Wachsmannova L, Adamcikova Z, Stevurkova V, Krcmery V, Zajac V. Microflora of intestinal and respiratory tract in AIDS process, J Antivir Antiretrovir. 2013; 15: 1-6.

[7] Hainova K, Adamcikova Z, Ciernikova S, Stevurkova V, Krcmery V, Zajac V. Detection of proteins homologous with HIV-1 antigens in bacteria of positive patients - phase II. Neuro Endocrinol Lett. 2014; 35: 101-106.

[8] Zajac V. The fundamental role of bacteria and yeasts in AIDS progression. J Vaccines Vaccin. $2014 ; 5: 4$.

[9] Wachsmannova L, Ciernikova S, Majek J, Mego M, Stevurkova V, Zajac V. Internalization property of intestinal bacteria in colon cancer and HIV/AIDS patients. Neuro Endocrinol Letters. 2016; 37: 245- 250.

[10] Zajac V. Evolutionary view of the AIDS process. J Int Med Res. Oct 2018; 46(10): 4032-4038.

[11] Vladimir Zajac. Can CCR5 delta 32 mutation be a proof of possible HIV participation in the Black Dead epidemic? Journal of Clinical Microbiology and Infectious Diseases. 2020; 3(3).

[12] Vladimir Zajac. Elimination ot the new coronavirus and the prevention of the second wave of infection. World Journal of Advances Research and Reviews. 2020: 08(01): 148-150.

[13] V Zajac. The Role of Bacteria in Viral Transmission. Japanesse Journal of Gastroenterology and Hepathology. 2020; 4(8): 1-2.

[14] Vladimir Zajac. An Overview on the Fundamental Role of Bacteria and Yeasts in AIDS Progression. Current Topics in Medicine and Medical Research. 2020; 3(2).

[15] Vladimir Zajac. The irreplaceable role of coronavirus carriers in its transmission and in second wave of infection. World Journal of Advanced Research and Reviews. 2021; 09(03): 226-228. 